\title{
Age-Related Changes in the Electrophysiologic Properties of the Atrium in Patients with no History of Atrial Fibrillation
}

\author{
Koichi SAKABE, ${ }^{1} \mathrm{MD}$, Nobuo FuKUdA, ${ }^{1} \mathrm{MD}$, Teru NADA, ${ }^{1} \mathrm{MD}$, \\ Hisanori SHINOHARA, ${ }^{1} \mathrm{MD}$, Yoshiyuki TAMURA, ${ }^{1} \mathrm{MD}$, \\ Tetsuzo WAKATSUKI, ${ }^{2}$ MD Akiyoshi NishIKADO,${ }^{2} \mathrm{MD}$, and Takashi OKI, ${ }^{2} \mathrm{MD}$
}

\section{SUMMARY}

Although atrial fibrillation is a common arrhythmia, especially in the elderly, little is known about age-related changes in the electrophysiologic properties of the atrium. The aim of this study was to analyze the effect of aging on atrial vulnerability to atrial fibrillation.

An electrophysiologic study was performed in 45 patients with no history of atrial fibrillation, Wolff-Parkinson-White syndrome, structural heart disease, or conditions with potential effects on cardiac hemodynamic or electrophysiologic function (15 females; mean age, $52 \pm 18$ years; range, 14 to 84 years). The following atrial excitability parameters were assessed: spontaneous or paced (A1) and extrastimulated (A2) atrial electrogram widths, percent maximum atrial fragmentation $(\mathrm{A} 2 / \mathrm{A} 1 \times 100)$, effective refractory period, wavelength index (ERP/A2), and inducibility of atrial fibrillation.

Atrial fibrillation was induced in 9 patients. Percent maximum atrial fragmentation was greater $(176 \pm 36$ vs $137 \pm 26 \%, P<0.001)$ and wavelength index was shorter $(2.4$ \pm 0.4 vs $3.2 \pm 0.9, P<0.01)$ in the patients with than without inducible atrial fibrillation. However, age was similar in patients with and without inducible atrial fibrillation (47 \pm 11 vs $53 \pm 19$ years, $P=0.36$ ). Percent maximum atrial fragmentation and effective refractory period directly correlated with age $(r=0.32, P<0.05$ and $r=0.45, P<0.001$, respectively). On the other hand, wavelength index $(3.1 \pm 0.9)$ did not correlate with age $(r=-0.05, P=0.77)$.

This study suggests that the mechanism triggering atrial fibrillation may be very well different between older and younger patients with atrial fibrillation, because younger patients have no marked substrate for atrial fibrillation. (Jpn Heart J 2003; 44: 385-393)

Key words: Aging, Electrophysiology, Wavelength

ATRIAL fibrillation is the most common arrhythmia encountered in clinical practice and is likely to become more common as the population ages. ${ }^{1,2)}$ How-

From ${ }^{1}$ Department of Cardiology and Clinical Research, National Zentsuji Hospital, Kagawa, ${ }^{2}$ Second Department of Internal Medicine, School of Medicine, University of Tokushima, Tokushima, Japan.

Address for correspondence: Koichi Sakabe, MD, Department of Cardiology and Clinical Research, National Zentsuji Hospital, 2-1-1, Senyu-cho, Zentsuji, Kagawa 765-8507, Japan.

Received for publication September 2, 2002.

Revised and accepted October 17, 2002. 
ever, all elderly people do not necessarily develop atrial fibrillation, and little is known about age-related changes in the electrophysiologic properties of the atrium. ${ }^{3,4)}$ Previous studies have shown that a short refractory period, conduction delay, and inducibility of atrial fibrillation characterize patients with paroxysmal atrial fibrillation, ${ }^{5-7)}$ but it remains unclear whether these indices merely represent the electrophysiologic nature of elderly people, irrespective of whether they have atrial fibrillation.

It is important to understand the relationship between atrial electrophysiologic properties and aging because these findings may help differentiate elderly people vulnerable to atrial fibrillation from others and identify the age at which people become vulnerable to atrial fibrillation. The purpose of this study was to analyze the effect of aging on atrial vulnerability in patients with no history of atrial fibrillation, Wolff-Parkinson-White syndrome, or structural heart disease.

\section{METHODS}

Patient population: Forty-five consecutive patients who underwent electrophysiologic studies for syncope or tachycardia at our institute and who did not meet exclusion criteria were enrolled in this study to examine the relationship between age and atrial vulnerability. All patients had a physical examination and underwent resting electrocardiogram, chest radiograph, echocardiogram, and routine hematologic and biochemical testing to exclude structural heart disease and conditions with potential effects on cardiac hemodynamic or electrophysiologic function. Patients were excluded from participation if they had prior documented atrial fibrillation, Wolff-Parkinson-White syndrome, structural heart disease, congestive heart failure, hypertension, diabetes, ionic disturbances in blood tests, or were smokers, because these factors are known to influence atrial vulnerability to atrial fibrillation. ${ }^{8-10)}$ Written informed consent was obtained from all subjects before enrollment. The study was carried out during routine electrophysiologic investigation, and, when necessary, before catheter ablation of the tachycardia.

Electrophysiologic studies: All antiarrhythmic drugs were discontinued prior to the studies for at least five times the half-life of each drug. Patients were studied in the fasting, nonsedated state. A 5 French quadripolar electrode catheter with 5mm spacing (DAIG) was inserted through the right femoral vein and positioned against the high right atrium. Atrial stimulation was delivered from the first pole, and atrial electrograms were recorded from the second pole. Before the stimulation procedure, $5000 \mathrm{IU}$ of heparin was injected intravenously, followed by an infusion of heparin at $1000 \mathrm{IU} /$ hour.

Stimulation was performed using square wave impulses 2-ms in duration at an intensity twice the threshold level, which were delivered by a programmable 
stimulator (SEC-3102, Nihon Kohden). Intracardiac electrograms were recorded (gain $2 \mathrm{mV} / \mathrm{cm}$, filter 30 to $500 \mathrm{~Hz}$ ) using an electrophysiologic recording system (Cardiolab; Prucka Engineering). To create the atrial vulnerability index, as described subsequently, ${ }^{1-4)}$ programmed electrical stimulation using eight basic stimuli at a cycle length of $500 \mathrm{~ms}$ followed by extrastimuli was delivered. Basic stimuli and the corresponding atrial deflection were designated $\mathrm{S} 1$ and A1, respectively. S2 and A2 were a single extrastimulus and the provoked atrial deflection. The S1-S2 interval was decreased by $10 \mathrm{~ms}$ until the extrastimulation failed to capture the atrium. In addition, programmed electrical stimulation with 2 extrastimuli (S2 and S3) was delivered to assess the inducibility of atrial fibrillation, when atrial fibrillation was not induced by a single extrastimulus.

Definition of the atrial vulnerability index: The following electrophysiologic indices of atrial vulnerability were measured and assessed regarding the relationship between age and atrial vulnerability.

1) Percent maximum atrial fragmentation: Percent maximum atrial fragmentation was defined as "the maximum width of A2 / width of A $1 \times 100$ ". The width of A2 was increased when the premature stimulation was given earlier in the cardiac cycle. The maximum width of A2 was, therefore, obtained at the shortest S1$\mathrm{S} 2$ interval that could provoke A2.,10-13)

2) Right atrial effective refractory period: Right atrial effective refractory period was defined as "the longest S1-S2 interval that does not provoke A2 in the high right atrium". ${ }^{10,13-15)}$

3) Wavelength index: Wavelength index was defined as "the ratio of effective refractory period / maximum width of A2". ${ }^{10,13-16)}$

4) Inducibility of atrial fibrillation: The inducibility of atrial fibrillation was assessed through the progressively aggressive stimulation protocol using single or double extrastimulation. Atrial fibrillation inducibility was defined as occurring when atrial fibrillation with a duration of at least 1 minute was induced. When atrial fibrillation persisted beyond 5 minutes, electrical cardioversion was performed. ${ }^{17)}$

Statistical analysis: Continuous data are expressed as the mean \pm SD and were analyzed by Student's $t$ test. Comparisons between clinical variables were evaluated by the chi-square test. Correlation coefficients for the relationship between two variables were derived using standard linear regression methods. A value of $P<0.05$ was considered statistically significant. 


\section{Results}

Patient characteristics: The study population consisted of 45 patients aged 14 to 84 years (mean, $52 \pm 18$ years) (Table I). Underlying arrhythmias were atrioventricular nodal reentrant tachycardia $(n=23)$, idiopathic ventricular tachycardia $(n$ $=6$ ), atrioventricular nodal block $(n=10)$, and neurally-mediated syncope ( $n=$ 6). The mean left atrial diameter was $33 \pm 6 \mathrm{~mm}$.

Atrial vulnerability index and age: Atrial fibrillation was induced in 9 patients (Table II). There was no difference between patients with and without inducible atrial fibrillation with respect to age ( $47 \pm 11$ vs $53 \pm 19$ years, $P=0.36$ ), gender, or left atrial diameter. In terms of electrophysiologic parameters, percent maximum atrial fragmentation was greater and the wavelength index was smaller in patients with than without inducible atrial fibrillation (176 \pm 36 vs $137 \pm 26 \%, P$ $<0.001$ and $2.4 \pm 0.4$ vs $3.2 \pm 0.9, P<0.01$, respectively), though the right atrial effective refractory period was similar in the two groups.

The percent maximum atrial fragmentation of all subjects ranged from 106 to $250 \%$ (mean, $145 \pm 32 \%$ ). Percent maximum atrial fragmentation had a positive correlation with age $(r=0.32, P<0.05)$. Right atrial effective refractory period ranged from 180 to $390 \mathrm{msec}$ (mean, $246 \pm 45 \mathrm{msec}$ ), and also had a positive correlation with age $(r=0.45, P<0.001)$. In contrast, the wavelength index, which ranged from 1.6 to 5.3 (mean, $3.1 \pm 0.9$ ), did not correlate with age $(r=$ $-0.05, P=0.77$ ) (Figure).

Table I. Demographics and Clinical Characteristics of the Study Population

\begin{tabular}{lc}
\hline No. of patients & 45 \\
Age (years) & $52 \pm 18(14$ to 84$)$ \\
Gender & $15(33 \%)$ \\
Women & $30(67 \%)$ \\
Men & \\
Arrhythmia & $23(52 \%)$ \\
AVNRT & $6(13 \%)$ \\
IVT & $10(22 \%)$ \\
AVB & $6(13 \%)$ \\
NMS & $33 \pm 6$ \\
LAD (mm)
\end{tabular}

Values are mean \pm SD or number of patients (\%). AVNRT $=$ atrioventricular nodal reentrant tachycardia; IVT = idiopathic ventricular tachycardia; AVB = atrioventricular nodal block; NMS = neurally-mediated syncope; LAD = left atrial diameter. 
Table II. Comparison of the Patients with and without Inducible Atrial Fibrillation

\begin{tabular}{lccc}
\hline & AF induction (+) & AF induction (-) & $P$ Value \\
\hline No. of patients & $9(20 \%)$ & $36(80 \%)$ & \\
Age (years) & $47 \pm 11$ & $53 \pm 19$ & NS \\
Gender & & & \\
$\quad$ Women/Men & $3 / 6$ & $12 / 24$ & NS \\
Atrial vulnerability index & & & \\
$\quad$ A1 (msec) & $56 \pm 15$ & $63 \pm 20$ & NS \\
$\quad$ Max A2 (msec) & $96 \pm 17$ & $85 \pm 28$ & NS \\
$\quad$ \%AF & $176 \pm 36$ & $137 \pm 26$ & $<0.001$ \\
$\quad$ ERP (msec) & $227 \pm 29$ & $251 \pm 47$ & NS \\
$\quad$ WLI & $2.4 \pm 0.4$ & $3.2 \pm 0.9$ & $<0.01$ \\
LAD (mm) & $36 \pm 7$ & $32 \pm 6$ & NS \\
\hline
\end{tabular}

Values are mean $\pm \mathrm{SD}$ or number of patients $(\%)$. $\mathrm{AF}=$ atrial fibrillation; \%MAF $=$ percent maximum atrial fragmentation; ERP = right atrial effective refractory period; WLI = wavelength index; $\mathrm{LAD}=$ left atrial diameter.
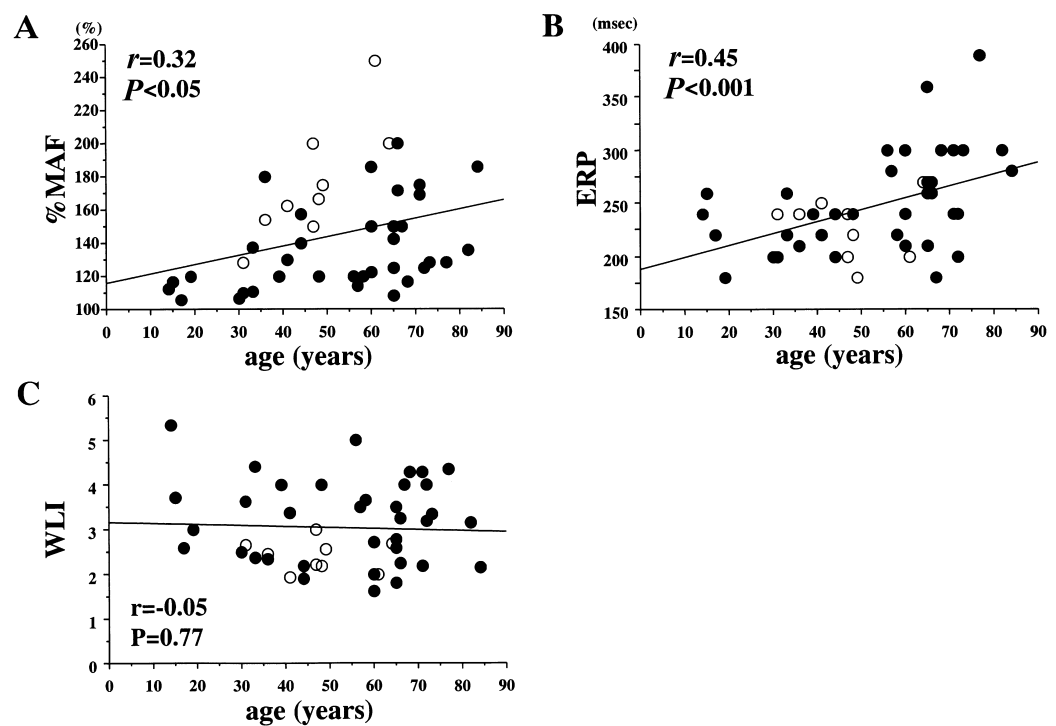

Figure. Correlation between age and percent maximum atrial fragmentation (\%MAF, A), right atrial effective refractory period (ERP, B), and wavelength index (WLI, C). Percent maximum atrial fragmentation and right atrial effective refractory period correlated directly with age, but wavelength index did not correlate with age. Open circles; patients with inducible atrial fibrillation, closed circles; patients without inducible atrial fibrillation.

\section{DISCUSSION}

Major findings: To our knowledge, this is the first study to analyze the effect of aging on the atrial vulnerability index in terms of factors such as the wavelength index and inducibility of atrial fibrillation in patients with no history of atrial 
fibrillation. Wavelength index did not correlate with age, and inducibility of atrial fibrillation was not a function of age. However, in this non-atrial fibrillation population, percent maximum atrial fragmentation was directly correlated with age and was smaller in the younger patients. Younger patients have no marked substrate for atrial fibrillation.

Atrial vulnerability index and aging: Atrial fibrillation is one of the most common supraventricular arrhythmias, especially in elderly people. ${ }^{1,2)}$ The prevalence of atrial fibrillation increases with age, and is $10 \%$ in the population $>70$ years old. ${ }^{2)}$ The atrial electrophysiologic properties of patients with atrial fibrillation have been studied, and the propensity for this arrhythmia has been expressed electrophysiologically as atrial vulnerability. Although several parameters, such as the atrial refractory period, fragmented atrial activity, atrial conduction delay, repetitive atrial firing, wavelength index, and inducibility of atrial fibrillation have been proposed as indices of atrial vulnerability, it remains uncertain whether these indices also represent inherent electrophysiologic properties in elderly people without atrial fibrillation. -7,10-17) $^{-1}$

The percent maximum atrial fragmentation has been reported to reflect prolongation of the atrial electrogram, the presence of a conduction abnormality, or the occurrence of atrial fibrillation. ${ }^{13)}$ In this study, the percent maximum atrial fragmentation was greater in patients with inducible atrial fibrillation than in those without inducible atrial fibrillation, which is concordant with previous reports. In addition, the percent maximum atrial fragmentation correlated positively with age and became greater in elderly people. These results suggest that the severity of the atrial conduction abnormalities is closely related to aging. This finding may be interpreted as evidence of the fact that aging induces structural changes in atrial muscle. ${ }^{18)}$ In terms of atrial conduction abnormalities, aging produces a progressive increase in the percent maximum atrial fragmentation that could be a predisposing factor for atrial fibrillation in this nonatrial fibrillation population.

Some previous studies have shown that a short refractory period can raise the likelihood that micro-reentry occurs, which characterizes the patients with paroxysmal atrial fibrillation. ${ }^{5,19)}$ On the other hand, no significant changes have been reported in the refractory period of diseased atrium. ${ }^{7,20)}$ We found no significant difference in the right atrial effective refractory period between patients with and without inducible atrial fibrillation, which is consistent with the latter results. A positive correlation existed between the right atrial effective refractory period and age in this study, and the right atrial effective refractory period became longer as age increased. Michelucci, et $a l^{3)}$ and Kavanagh, et $a l^{4)}$ also found a similar positive correlation between the right atrial effective refractory period and age. In addition, it has been suggested that this age-related prolongation of right 
atrial effective refractory period may be affected by age-related changes in the action potential duration, resting membrane potential, and autonomic nervous system. ${ }^{21-23)}$ These results indicate that aging may prolong the right atrial effective refractory period. However, prolongation of the right atrial effective refractory period could not have affected the inducibility of atrial fibrillation, because no difference in the right atrial effective refractory period was observed in patients with and without inducible atrial fibrillation.

We also evaluated the wavelength index as an atrial vulnerability index. The wavelength has been defined as the distance traveled by the depolarization wave during the refractory period. ${ }^{24)}$ Accordingly, Attuel, et al ${ }^{16)}$ reported that the wavelength index represented the minimum length of the reentrant circuit at that site. Some previous studies found that a shorter wavelength index indicated greater vulnerability to atrial fibrillation. ${ }^{10,14,16)}$ In this study, the wavelength index was shorter in patients with inducible atrial fibrillation than in those without inducible atrial fibrillation, which is consistent with these previous reports. However, we found no correlation between the wavelength index and age, which is the first time this finding has been described. The wavelength index can be divided into two components: the conduction velocity and the refractory period. ${ }^{24)} \mathrm{We}$ found that aging introduces atrial conduction abnormalities and prolongs refractoriness. The age-related changes in these parameters may reduce the effect of aging on wavelength index, because longer conduction delays are associated with longer refractoriness. In addition, the failure to observe age-related changes in the induction of atrial fibrillation in our patients with no history of atrial fibrillation suggested that age-related changes in the wavelength index do not exist. These data suggest that not only conduction abnormalities but other factors as well may affect the inducibility of atrial fibrillation.

Study limitation: Patients with Wolff-Parkinson-White syndrome were excluded from participation in our study, because branched accessory pathways causing microreentry have been suggested as causative factors for atrial fibrillation. ${ }^{10}$ ) Nevertheless, the patients in this study had different types of arrhythmias or had neurally-mediated syncope. Although the effects of the underlying diseases on atrial vulnerability might not be negligible, this is a limitation common to many studies. ${ }^{714,16)}$ In this study, we found no difference with respect to age between patients with and without inducible atrial fibrillation, which is not concordant with previous reports. ${ }^{1,2)}$ The reason for this discrepancy might be due to the underlying arrhythmias.

The sample size of the study population was small because of the exclusion criteria, and the relationship between our electrophysiologic findings and prognosis remains unclear. To clarify the clinical significance of our electrophysiologic 
findings, a long-term, prospective follow-up study involving a larger number of patients is necessary.

Conclusions: In this non-atrial fibrillation population, percent maximum atrial fragmentation was directly correlated with age and was larger in the elderly, though the wavelength index and the inducibility of atrial fibrillation were not affected by aging. Considering these findings, we conclude that the mechanism triggering atrial fibrillation may very well be different between older and younger patients with atrial fibrillation, because younger patients have no marked substrate for atrial fibrillation.

\section{REFERENCES}

1. Furberg CD, Psaty BM, Manolio TA, Gardin JM, Smith VE, Rautaharju PM. Prevalence of atrial fibrillation in elderly subjects (the Cardiovascular Health Study). Am J Cardiol 1994; 74: 236-41.

2. Kannel WB, Abbott RD, Savage DD, McNamara PM. Coronary heart disease and atrial fibrillation: The Framingham Study. Am Heart J 1983; 106: 389-96.

3. Michelucci A, Padeletti L, Fradella GA, et al. Aging and atrial electrophysiologic properties in man. Int J Cardiol 1984; 5: 75-81.

4. Kavanagh KM, Wyse DG, Mitchell LB, Duff HJ. Cardiac refractoriness: age-dependence in normal subjects. J Electrocardiol 1989; 22: 221-5.

5. Cosio FG, Palacios J, Vidal JM, Cocina EG, Gomez-Sanchez MA, Tamargo L. Electrophysiologic studies in atrial fibrillation. Slow conduction of premature impulses: A possible manifestation of the background for reentry. Am J Cardiol 1983; 51: 122-30.

6. Shimizu A, Fukatani M, Tanigawa M, Mori M, Hashiba K. Intra-atrial conduction delay and fragmented atrial activity in patients with paroxysmal atrial fibrillation. Jpn Circ J 1989; 53: 1023-30.

7. Inoue K, Shirayama T, Shiraishi H, et al. Clinical significance of the atrial fibrillation threshold in patients with paroxysmal atrial fibrillation. PACE 2001; 24: 796-805.

8. Benjamin EJ, Levy D, Vaziri SM, D'Agostino RB, Belanger AJ, Wolf PA. Independent risk factors for atrial fibrillation in a population-based cohort. The Framingham Heart Study. JAMA 1994; 271: 840-4.

9. Psaty BM, Manolio TA, Kuller LH, et al. Incidence of and risk factors for atrial fibrillation in older adults. Circulation 1997; 96: 2455-61.

10. Niwano S, Yamaura M, Kitano Y, et al. Importance of retrograde atrial activation in atrial fibrillation genesis in the initiation of atrial fibrillation in Wolff-Parkinson-White syndrome. Jpn Heart J 1999; 40: 281-93.

11. Ohe T, Matsuhisa M, Kamakura S, et al. Relation between the widening of the fragmented activity-zone and atrial fibrillation. Am J Cardiol 1983; 52: 1219-22.

12. Niwano S, Aizawa Y. Fragmented atrial activity in patients with transient atrial fibrillation. Am Heart J 1991; 121: $62-7$.

13. Tanigawa M, Fukatani M, Konoe A, Isomoto S, Kadena M, Hashiba K. Prolonged and fractionated right atrial electrograms during sinus rhythm in patients with paroxysmal atrial fibrillation and sick sinus node syndrome. $\mathrm{J}$ Am Coll Cardiol 1991; 17: 403-8.

14. Padeletti L, Michelucci A, Giovannini T, et al. Wavelength index at three atrial sites in patients with paroxysmal atrial fibrillation. PACE 1995; 18: 1266-71.

15. De Sisti A, Leclercq JF, Fiorello P, Manot S, Halimi F, Attuel P. Electrophysiologic characteristics of the atrium in sinus node dysfunction: Atrial refractoriness and conduction. J Cardiovasc Electrophysiol 2000; 11: 30-3.

16. Attuel P, Pellerin D, Gaston J. Latent atrial vulnerability: New means of electrophysiologic investigations in paroxysmal atrial arrhythmia. In: Attuel P, Coumel P, Janse M, editors The Atrium in Health and Disease. Mount Kisco. New York: Futura Publishng Co., Inc., 1989; 159-200. 
17. Ramanna H, Hauer RN, Wittkampf FH, et al. Identification of the substrate of atrial vulnerability in patients with idiopathic atrial fibrillation. Circulation 2000; 101: 995-1001.

18. Spach MS, Dolber PC. Relating extracellular potential and their derivates to anisotropic propagation at a microscopic level in human cardiac muscle: Evidence for electrical uncoupling of side-to-side fiber connections with increasing age. Circ Res 1986; 58: 356-71.

19. Moe GK, Rheinbolt WC, Abildskov JA. A computer model of atrial fibrillation. Am Heart J 1964; 67: 200-20.

20. Simpson RJ Jr, Amara I, Foster JR, Woelfel A, Gettes LS. Thresholds, refractory periods, and conduction times of the normal and diseased human atrium. Am Heart J 1988; 116: 1080-90.

21. Toda N. Age-related changes in the transmembrane potential of isolated rabbit sino-atrial nodes and atria. Cardiovasc Res 1980;14: 58-63.

22. Sachs C, Hamberzer B, Kayser L. Cardiovascular responses and plasma catecholamines in old age. Clin Physiol 1985; 5: 553-65.

23. Brodde OE, Anlauf M, Graben N, Bock KD. In vitro and in vivo down-regulation of human platelet alpha 2adrenoceptors by clonidine. Eur J Clin Pharmacol 1982; 23: 403-9.

24. Wiener N, Rosenblueth A. The mathematical formulation of the problem of conduction of impulses in a network of connected excitable elements, specifically in cardiac muscle. Arch Inst Cardiol Mex 1946; 16: 205-65. 